\title{
EVOLUTION OF THERMALLY GENERATED NEUTRON-STAR MAGNETIC FIELDS
}

\author{
ROGER W. ROMANI \\ Institute for Advanced Study \\ LARS E. HERnQuist \\ Princeton University
}

\begin{abstract}
Strongly magnetized neutron stars are believed to underlie a variety of astrophysical systems, although conflicting observational and theoretical evidence has led to debate on the origin and stability of these magnetic fields. Here we describe a new model of neutron star magnetic moments, assuming that the fields are generated at birth and following their evolution to ages as large as the Hubble time. With realistic thermal evolution and conductivities, isolated neutron stars will maintain large magnetic dipole fields. As suggested elsewhere field modification under mass accretion might lead to torque decay. We identify an operative mechanism for this process; the results of this unified picture are in agreement with observations of a wide range of neutron star systems.
\end{abstract}

Newborn neutron stars are thought to have large dipole surface fields, as evidenced by the spindown torques on young radio and $\mathrm{X}$-ray pulsars. For a radio pulsar of period $P$ spinning down in isolation, magnetic dipole braking models give an estimate of the field

$$
B_{\mathrm{s}} \approx\left(\frac{3 I c^{3} P \dot{P}}{8 \pi^{2} R^{6}}\right)^{1 / 2} \approx 1.0 \times 10^{12}\left(P \dot{P}_{-15}\right)^{1 / 2} \mathrm{G}
$$

where the period derivative is $10^{-15} \dot{P}_{-15} \mathrm{~s} \mathrm{~s}^{-1}$. For the bulk of known radio pulsars, the estimated surface field [eq.(1)] lies near $10^{12.5} \mathrm{G}$, with a dispersion (Narayan and Ostriker 1990) $\sim 10^{0.3} \mathrm{G}$. The origin of this field is a subject of long-standing dispute; a popular scenario involves flux freezing and amplification of interior fields of the progenitor object, which leaves the above characteristic value unexplained. Alternatively, it has been proposed that magnetic fields can be generated in young stars by thermomagnetic effects (Blandford, Applegate, and Hernquist 1983).

The torque appears to decay on a timescale of several million years, leading to a rapid disappearance of the pulsars from the radio sky (Lyne, Manchester, and Taylor 1985, Blandford, Applegate, and Hernquist 1983). Recent observational and theoretical work has, however, clouded this picture appreciably. The binary and millisecond radio pulsars, in particular, make it clear that the fields of some neutron stars can be both a factor $\sim 10^{4}$ lower than the canonical birth value and remain stable (Kulkarni 1986) for $\gtrsim 10^{9} \mathrm{yr}$. Conversely, some $\gamma$ ray burst sources, believed to be old neutron stars, have been shown (Murakami et al. 1988) to have high surface fields $\gtrsim 10^{12} \mathrm{G}$; in addition, theoretical arguments (Baym, Pethick, and Pines 1969, Sang and Chanmugam 1987) imply that it is difficult to reduce the magnetic flux threading much of the neutron-star interior.

We present here a simple picture of magnetic field evolution that explains the above behavior. Our ansat $z$ is that the field is generated after neutron star birth during the initial cooling phase, either by thermomagnetic effects in the solid crust (Blandford, Applegate, and Hernquist 1983) or via dynamo processes in the still-liquid envelope above the solid interior. Thermomagnetic generation is most effective where the temperature gradient is steepest, i.e., at the lowest solid densities. Similarly, dynamo-generated flux will be frozen into the star at the base of the liquid sea. Thus the yield stress of the lattice at the melt surface provides a maximum value for the local dipolar field that can be frozen into this outer crust. We shall assume that the (unprescribed) mechanism of field generation is effective, saturating at this critical value; higher fluxes will break the coulomb lattice and escape from the star. Balancing the magnetic stress with the yield stress of the lattice determined from the critical stain angle $\epsilon$ and the shear modulus $\mu$, we find that this critical field is

$$
B_{\mathrm{c}}=(8 \pi \epsilon \mu)^{1 / 2}=3.7 \times 10^{13} \epsilon_{-2}^{1 / 2} Z A^{-2 / 3} \rho_{11}^{2 / 3} \text {. }
$$

As the star cools, the melt surface moves out to lower densities; when the underlying field dominates resistance to motions of the melt surface at $\rho \sim 10^{7} \mathrm{~g} \mathrm{~cm}^{-3}$, the addition of flux to the solid will cease.

Results of a cooling calculation for a $1.4 M_{\odot}$, 
$R_{*}=10.9 \mathrm{~km}$, FP model star presented by Tsuruta (1987) are taken as a typical example of the neutron-star thermal evolution. Using the analytic results of Hernquist and Applegate (1984), we obtain the temperature run in the solid crust between the core (isothermal below neutron drip) and the melt surface at

$$
T_{\mathrm{m}}=\frac{Z^{2} e^{2}}{k \Gamma_{\mathrm{m}}}\left(\frac{4 \pi \rho}{3 m_{\mathrm{p}} A}\right)^{1 / 3}
$$

Here $\Gamma_{\mathrm{m}} \approx 160$, and the local composition $Z$ and $A$ are determined from the BPS results (Baym, Pethick, and Sutherland 1971) for $\rho \lesssim$ $2 \times 10^{11} \mathrm{~g} \mathrm{~cm}^{-3}$, and from the BB computations (Buchler and Barkat 1971) at higher densities. At late times we include interior vortex creep heating (Shibazaki and Lamb 1989) which generates a surface temperature $T_{\mathrm{s}} \approx 2.9 \times$ $10^{4}\left(I_{\mathrm{c}, 43} \omega_{\mathrm{cr},-2} \dot{P}_{-15} P^{-2}\right)^{1 / 4} \mathrm{~K}$, where the crustal moment of inertia and critical slip velocity are $10^{43} I_{\mathrm{c}, 43} \mathrm{~g} \mathrm{~cm}^{2}$ and $10^{-2} \omega_{\mathrm{cr},-2} \mathrm{~s}^{-1}$, respectively.

The field held in the solid crust will undergo slow ohmic diffusion (Ostriker and Gunn 1969). We are interested only in the dipole component of the anchored field, so we follow Sang and Chanmugam by taking the field decay in the solid, where internal motions are negligible, to be described by

$$
\frac{\partial g}{\partial t}=\frac{c^{2}}{4 \pi R_{*}^{2} \sigma(r, t)}\left(\frac{\partial^{2} g}{\partial^{2} r}-\frac{2}{r^{2}} g\right) .
$$

Here $g(r, t)=r A_{\phi} / \sin (\theta)$ is the one dimensional 'vector potential', depending only on radius, which completely describes the dipole field distribution. The perpendicular magnetic moment is given by $m_{\mathrm{z}}=3 /(8 \pi) \int_{R_{\mathrm{*}}} \mathbf{B}_{\mathrm{z}} d^{3} r=g\left(R_{*}\right) R_{*}=B_{\mathrm{s}} / R_{*}^{2}$.

There are two important contributions to the resistivity of the neutron-star crust. The first is scattering of electrons by phonons in the Coulomb lattice. We compute this conductivity at each depth and temperature by interpolating the numerical results of Flowers and Itoh (1976), reduced by $\sim 3$ to bring them into agreement with the more limited computations of Yakovlev and Urpin (1980). This will be the dominant source of resistivity at high temperatures and low densities. At late times, however, scattering by a lattice impurity fraction $\chi$ will dominate, leading to a conductivity of $\sigma_{\text {imp }}=$ $2 \times 10^{23}\left(Z \rho_{11} / A\right)^{1 / 3} Z /\left(\chi \Delta Z^{2}\right) \mathrm{s}^{-1}$, independent of the temperature. Simple estimates (Flowers and Ruderman 1977) suggest $\chi \Delta Z^{2} \sim 10^{-3}$ for crustal matter; we adopt this value as fiducial. These conductivities add harmonically in the crust.

A model star is constructed by integrating the Oppenheimer-Volkoff equation with our adopted equation of state to get $\rho(z)$. With $T(t)$ and $\sigma(t)$ as above, we evolve the model, following the growth and ohmic diffusion of $g$, solving eq.(3) by a forward-backwards symmetric Crank-Nicholson scheme. During the growth phase the field at the melt is held to the saturation value $B_{c}$; after growth ceases the boundary condition excludes surface currents. At all times the field was held to be less than the local $B_{c}$ throughout the star; excess flux was presumed to break the lattice, advect to the surface and disappear. Details of the computations appear elsewhere (Romani 1990b).

The result is shown by the heavy line in figure 1. The computation starts at $t_{0}=0.2 \mathrm{yr}$ after neu-

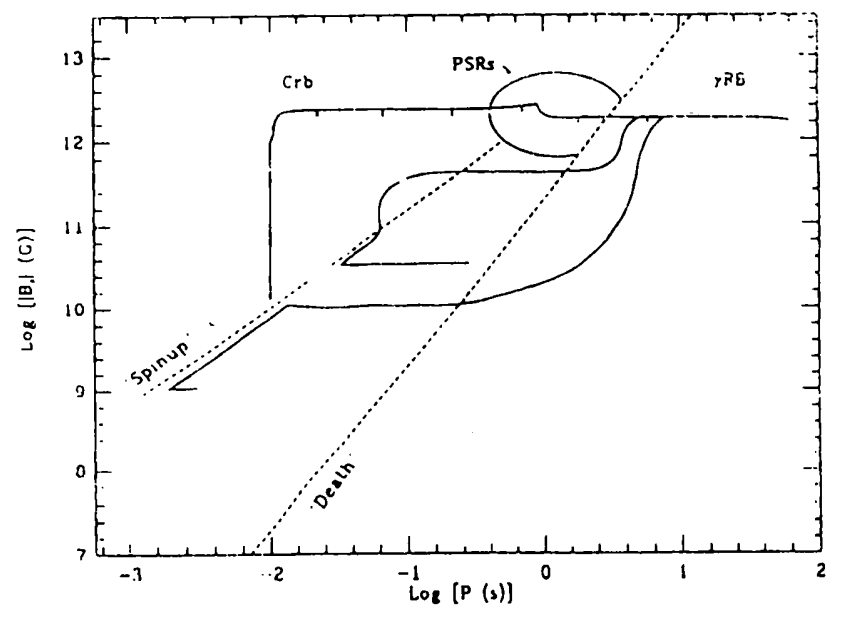

Figure 1 Magnetic field vo. spin period. The heavy line gives the track for an isolated star, with tick marks spaced as $\log (t)$. Thin lines sketch the evolution of accreting systems; observed locations of various types of neutron stars are noted.

tron star birth, when the melt surface has reached neutron drip and the lattice is fully formed. The field grows quite rapidly, reaching $10^{12} \mathrm{G}$ after only a few years. Our simple prescription for the initial conditions produce a typical neutron-star field quite well. The growth behavior is not very sensitive to $t_{0}$-maximum fields are $\sim 10^{12} \mathrm{G}$ for $t_{0}$ as large as $10 \mathrm{yr}$. However, dependence on the critical strain angle $\left(\epsilon^{1 / 2}\right)$, stellar radius, and moment of inertia are adequate to explain the observed range of $B$ values.

Growth ceases at $\sim 10^{\circ}$ years, and the field decays ohmically in the outermost crust on a timescale $\sim 10^{6} \mathrm{yr}$. This partial screening of the interior flux gives a $\sim 30 \%$ decrease in $B_{8}$. However, at these times the star is sufficiently cold that thereafter flux is well frozen into the crust. Only a slight decay on a timescale $\gtrsim 10^{10} \mathrm{yr}$ is apparent due to the impurity scattering resistivity. Note that we have loaded the outer crust with the maximum allowed field; other initial conditions should produce configurations with currents held deeper in the star and longer ohmic decay times. Thus our conclusion is quite robust-with realistic temperatures and conductivities an initial magnetic field can decay ohmi- 
cally by only a modest factor.

Figure 1 shows the evolution in the $P$ vs. $B$ diagram, when the spin period is $10 \mathrm{~ms}$ at $t_{0}$ (full line). The star moves rapidly to the region in which young pulsars, such as Crab and Vela are found. As the track evolves to longer periods there is still a modest increase in $\dot{P}$ for the young objects, corresponding to 'braking indices' less than 3 , as observed for several such pulsars (Blandford and Romani 1988). After joining the bulk of the observed pulsars, at $P \sim 1 \mathrm{~s}, B \sim 10^{12} \mathrm{G}$, the track drops slightly in $B_{\mathrm{s}}$ and crosses the so-called 'death line'. This decrease can help to explain the observed torque decay of radio pulsars, possibly abetted by additional resistivity in the surface layers and alignment of the magnetic and spin axes. After disappearing from the radio sky, the pulsars continue to spin down, reaching $P \gtrsim 30 \mathrm{~s}$ in a Hubble time. The parameters in this pulsar graveyard accord well with the limited observations of $\gamma$-ray burster magnetic fields (Murakami et al. 1988) and spin periods (e.g. GRB030579).

It has been noted (Taam and van den Heuvel 1986, Bailes 1989) that all neutron stars with anomalously low magnetic moments are in mass transfer binaries or resided in such systems in the past. Contact binaries produce the 'recycled' binary millisecond and globular cluster pulsars. There is even evidence that the magnetic moments of these objects are correlated with the duration of the mass accretion phase or the total amount of matter accreted (Taam and van den Heuvel 1986, de Kool and van Paradijs 1986, Romani 1990a). In the past, these discussions appealed to either an inverse battery effect (Blondin and Freese 1986) or an accretion-induced collapse origin of the resultant pulsars, both of questionable efficacy.

Having computed our model's long-lived residual field, we are now in a position to describe its subsequent modification under mass accretion. There are two principal effects. First, the energy released during accretion will reheat the outer layers of the neutron star to $T_{\mathrm{h}} \sim\left[G M_{*} \dot{M} /\left(4 \pi R_{*}^{3} \sigma_{B}\right)\right]^{1 / 4}$, lowering the conductivity and hastening ohmic decay. Far more important, however, is the compression of the outer layers of the star due to the accreted overburden and the inward advection of mag- netic field-bearing crust. Matter flowing onto the polar cap is channeled by the magnetic field, building up until the static pressure is comparable to $B^{2} / 8 \pi$. At this point material will begin to flow sideways. For strong fields the magnetic pole confines the flow to densities $\rho \gtrsim 10^{6} \mathrm{~g} \mathrm{~cm}^{-3}$, where the field is relatively well coupled to the matter. Thus the spreading material will decrease the density of field lines at the magnetic pole, carrying them towards the equator, where they will be advected below the surface and trapped in the underlying crust. As the polar-cap field decreases, the matter will tend to leak out closer to the surface, at lower densities. For surface fields of $\sim 10^{8} \mathrm{G}$ the accretion column will disrupt at densities of a few, where the ohmic decay and fluid interchange timescales will be shorter than the flow timescale. Thus material will no longer drag the magnetic flux lines beneath the surface, although it will continue to advect the current carrying layers deeper within the star. The resultant asymptotic value of the field will depend on the total amount of mass accreted, and through the surface temperature reheating, upon the accretion rate.

In figure 1 we sketch tracks of the resultant field and spin evolution. The accreted matter adds angular momentum (Ghosh and Lamb 1979), moving the star to smaller $P$. Systems with high $\dot{M}$ and short accretion lifetimes (such as wide LMXB and common envelope systems) will suffer limited field reduction, resulting in systems such as PSR 0820+02, PSR 0655+64 and PSR 1913+16. Binaries with longer lived accretion phases and lower rates of mass transfer will result in neutron stars substantially augmented above their $\sim 1.4 M_{\odot}$ birth mass with fields decreased to near the asymptotic value above. The result should be recycled objects such as PSR 1953+10 and PSR $1620+21$, lying close to the 'spinup line'. Note finally, that for both types of pulsars the currents and fields have been carried to the high conductivity interior, precluding additional decay of $B_{s}$, a further virtue of our model.

Acknowledgment: RWR is supported by NSF grant PHY86-20266 and a grant from the Corning Glass Works. 